\title{
Equilibrium States for a One Dimensional Lattice Gas
}

\author{
H. J. BRASCAMP \\ Institute for Theoretical Physics, Rijks-Universiteit, Groningen, The Netherlands
}

Received November 11, 1970

\begin{abstract}
The thermodynamic equilibrium state can be defined directly for an infinite system via an equilibrium condition or via the variational principle. Both definitions are used to calculate the equilibrium state for a one dimensional lattice gas with finite range interactions.
\end{abstract}

\section{Introduction}

In this paper, the equilibrium state for a one dimensional classical lattice gas with finite range interactions will be calculated explicitly. As a starting point, two different definitions for an equilibrium state for an infinite system will be taken. Usually, the equilibrium state for given temperature and chemical potential is found as the limit of finite volume Gibbs states. Because the limit may not be unique, it may be useful to define the notion of equilibrium state directly for the infinite system. There are two ways known.

1. The Variational Principle. An equilibrium state is a translationally invariant state, that maximizes the pressure [1]. By the occurrence of the mean entropy, translations play an essential role.

2. A number of equivalent equilibrium conditions. A set of equations is given, solutions of which are by definition equilibrium states. Examples are the KMS condition [2,3], and the condition given by Dobrushin $[5,6]$ and Lanford and Ruelle [4]. For classical lattice systems, both conditions can be proved to be equivalent [7].

For the classical lattice gas, there is the following connection between the variational method and the equilibrium condition. If the interactions are translationally invariant, an invariant state satisfies the equilibrium condition precisely if it maximizes the pressure [4].

In the present paper, the above ideas will be applied to the one dimensional classical lattice gas with finite range, translationally invariant interactions. It is well known, that no phase transitions occur in this case [21]. 
In Section 4, the equilibrium state is calculated, starting from the equilibrium condition. As it should be, it proves to be unique and translationally invariant. It follows, that the variational principle must lead to the same state. However, since the calculation is quite different and interesting in itself, it will be given in Section 5 .

In Section 2, the necessary preliminaries are given. The partition function is rewritten with the help of the well known matrix method in Section 3.

\section{Definitions}

In the present model, each point of a $v$-dimensional lattice $Z^{v}$ can be in two states. We will concentrate on the lattice gas, where each point can be empty or occupied with one particle. Mathematically equivalent is the Ising model, where each point contains a molecule with "spin" up or down.

In this paper, we use the mathematical framework introduced by Ruelle, Gallavotti and Miracle [1,8-10]. We will briefly summarize the necessary preliminaries. In Section 2.2 and 2.3 some special attention will be payed to the one dimensional lattice.

\subsection{States}

A configuration of the lattice is determined by specifying for each lattice point, whether or not it contains a particle. So to a configuration there corresponds a subset $X$ of the lattice, viz. the set of occupied points.

For the definition of observables and states, see [1,7-10]. For the present paper, the following notion of state will be sufficient. A state is a set of non-negative quantities

$$
\left\{\mu_{\Lambda}(X): \Lambda \text { finite, } X \subset \Lambda\right\},
$$

satisfying the consistency relations

$$
\begin{gathered}
\sum_{X \subset A} \mu_{\Lambda}(X)=1 \\
\sum_{Y \subset M / A} \mu_{M}(X \cup Y)=\mu_{\Lambda}(X) .
\end{gathered}
$$

The quantity $\mu_{\Lambda}(X)$ is interpreted as the probability, that the lattice points in $X$ are occupied, whereas the points of $\Lambda / X$ are empty. This interpretation leads to the relations (1). It follows, that the state is already determined by its correlation functions

$$
\varrho(X)=\mu_{X}(X)
$$


for finite $X \neq \emptyset$. An arbitrary $\mu_{A}(X)$ is expressed into correlation functions according to $[10]$

$$
\mu_{\Lambda}(X)=\sum_{Y \subset \Lambda / X} \varrho(X \cup Y)(-1)^{N(Y)},
$$

where $N(Y)$ is the number of points in $Y$.

\subsection{Translations}

A $v$-dimensional lattice is mapped onto itself by translation over any lattice vector $a$. The configuration $X$ is then carried over into the configuration $X+a$. A state is translationally invariant if

or

$$
\begin{gathered}
\mu_{\Lambda+a}(X+a)=\mu_{\Lambda}(X), \\
\varrho(X+a)=\varrho(X) .
\end{gathered}
$$

Let us now consider the one dimensional lattice. A finite volume will often be an interval of the form $[1, k]$. For notational convenience we will use the abbreviation

$$
\mu_{[1, k]}(X) \equiv \mu_{k}(X) .
$$

A state will be called invariant in the interval $[1, n]$ if

$$
\mu_{\Lambda}(X)=\mu_{\Lambda+1}(X+1)
$$

for any $\Lambda \subset[1, n-1], X \subset \Lambda$.

Let a set of $2^{n}$ numbers

$$
\mu_{n}(X), X \subset[1, n],
$$

be given. They define an invariant state in $[1, n]$ if and only if

$$
\begin{aligned}
\sum_{X \subset[1, n]} \mu_{n}(X) & =1 \\
\mu_{n}(X)+\mu_{n}(X \cup\{n\}) & =\mu_{n}(X+1)+\mu_{n}(\{1\} \cup(X+1)), X \subset[1, n-1] .
\end{aligned}
$$

The last equation is equivalent to Eq. (5) for $\Lambda=[1, n-1]$; cf. Eqs. (1). For an arbitrary $\Lambda \subset[1, n-1]$, Eq. (5) can be derived by repeated application of Eqs. (6).

Finally, a set of $2^{n}$ numbers $\mu_{n}(X), X \subset[1, n]$, and a set of $2^{n-1}$ numbers $\mu_{n-1}(X), X \subset[1, n-1]$, define an invariant state in $[1, n]$ if and only if

$$
\begin{array}{ll}
\sum_{X \subset[1, n]} \mu_{n}(X)=1 ; \quad \sum_{X \subset[1, n-1]} \mu_{n-1}(X)=1 & \\
\mu_{n-1}(X)=\mu_{n}(X)+\mu_{n}(X \cup\{n\}), & X \subset[1, n-1], \\
\mu_{n-1}(X)=\mu_{n}(X+1)+\mu_{n}(\{1\} \cup(X+1)), & X \subset[1, n-1] .
\end{array}
$$




\subsection{Interactions}

To a set $X$ of $n$ occupied lattice points there corresponds an $n$-body potential $\Phi(X)$, so that the total energy of a finite set $X$ of occupied points is

$$
U(X)=\sum_{Y \subset X} \Phi(Y) .
$$

The interaction $\Phi$ usually has the properties $[1,4]$

1. $\Phi(\emptyset)=0$

2. $\Phi(X+a)=\Phi(X)$

3a. $\|\Phi\|_{1} \equiv \sum_{0 \in X}|\Phi(X)| N(X)^{-1}<\infty$

3b. $\quad\|\Phi\|_{2} \equiv \sum_{0 \in X}|\Phi(X)|<\infty$.

The norm $\|\Phi\|_{1}$ plays a role in connection with the variational principle $[1,10]$. The norm $\|\Phi\|_{2}$, which is greater, naturally arises when time translations and equilibrium conditions are considered $[4,7]$.

One introduces the observable

$$
A_{\Phi}(X)=\sum_{\substack{Y \subset X \\ 0 \in Y}} \frac{\Phi(Y)}{N(Y)},
$$

which is interpreted as the contribution of the origin to the total energy of the configuration $X$. The expectation $\omega\left(A_{\Phi}\right)$ for an invariant state $\omega$ is then the mean energy per lattice site.

We now consider a one dimensional lattice with interactions of range $n$. That is, from

$$
0 \in X, \quad \Phi(X) \neq 0
$$

it follows that

$$
X \subset[-n+1, n-1] .
$$

Then the conditions $3 \mathrm{a}, \mathrm{b}$ of Eqs. (9) are automatically fulfilled. Also, $A_{\Phi}$ is a local observable in the interval $[-n+1, n-1]$, so

$$
\omega\left(A_{\Phi}\right)=\sum_{X \subset[-n+1, n-1]} \mu_{[-n+1, n-1]}(X) A_{\Phi}(X) .
$$

Another expression, which will be of special use for our purposes, is

$$
\omega\left(A_{\Phi}\right)=\sum_{X \subset[1, n]} \mu_{n}(X)\left[U(X)-\frac{1}{2} U\left(X^{(1)}\right)-\frac{1}{2} U\left(X^{(n)}\right)\right],
$$


where

$$
X^{(1)}=X \cap[2, n] ; \quad X^{(n)}=X \cap[1, n-1] .
$$

With Eqs.(1), (8), and (10), one easily proves that both (11) and (12) equal the expression

$$
\sum_{\substack{X \subset[1, n] \\ 1 \in X}} \varrho(X) \Phi(X) .
$$

\section{The Partition Function}

In statistical mechanics, one is generally interested in the grand canonical partition function of a finite volume $\Lambda$,

$$
Z_{\Lambda}=\sum_{X \subset A} \exp [-U(X)]
$$

The chemical potential $\mu$ is absorbed in the interaction as the one body potential, thus giving rise to a term $-N(X) \mu$ in the energy $U(X)$. The inverse temperature $\beta$ has been absorbed as a factor in the interaction.

The thermodynamic pressure is now found by

$$
P=\lim _{\Lambda \rightarrow \infty} \frac{1}{N(\Lambda)} \log Z_{\Lambda} .
$$

The existence of the limit has been proved for the classical lattice gas by Gallavotti and Miracle [8].

Let us return to the one dimensional lattice with interactions of range $n$. Then the partition function can be rewritten with the help of the matrix method $[11,12]$. Take an interval

$$
M=[1, m(n-1)],
$$

and let $X \subset M$. Because of the range $n$ of the interaction,

$$
\begin{gathered}
U(X)=U\left(X_{1} \cup X_{2}\right)-U\left(X_{2}\right)+U\left(X_{2} \cup X_{3}\right) \\
\cdots-U\left(X_{m-1}\right)+U\left(X_{m-1} \cup X_{m}\right),
\end{gathered}
$$

where

So

$$
X_{j}=X \cap[(j-1)(n-1)+1, j(n-1)] .
$$

$$
\begin{aligned}
Z_{M} & =\sum_{X_{1}, \ldots, X_{m}} \exp \left[-U\left(X_{1}\right)\right] \prod_{j=1}^{m-1} \exp \left[-U\left(X_{j} \cup X_{j+1}\right)+U\left(X_{j}\right)\right] \\
& =\sum_{X, X^{\prime}} \exp [-U(X)] R^{m-1}\left(X, X^{\prime}\right)
\end{aligned}
$$


where the $2^{n-1} \times 2^{n-1}$ matrix $R$ is given by

$$
R\left(X, X^{\prime}\right)=\exp \left[-U\left(X \cup X^{\prime}\right)+U(X)\right] .
$$

In Eqs. (15) and (16), $X$ is a subset of an interval of $n-1$ points, and $X^{\prime}$ is a subset of another such interval, immediately to the right of the first one. Due to the invariance of the interaction, $R$ is independent of the precise position of the intervals.

Another expression for $Z_{M}$ is

$$
\begin{aligned}
Z_{M} & =\sum_{X_{1}, \ldots, X_{m}} \exp \left[-U\left(X_{m}\right)\right] \prod_{j=1}^{m-1} \exp \left[-U\left(X_{j} \cup X_{j+1}\right)+U\left(X_{j+1}\right)\right] \\
& =\sum_{X, X^{\prime}} \exp [-U(X)] L^{m-1}\left(X, X^{\prime}\right)
\end{aligned}
$$

where

$$
L\left(X, X^{\prime}\right)=\exp \left[-U\left(X^{\prime} \cup X\right)+U(X)\right] .
$$

In Eqs. (17) and (18), $X^{\prime}$ is on the left of $X$, so in general the matrices $R$ and $L$ will differ. Introduce the matrices

$$
\begin{aligned}
r\left(X, X^{\prime}\right) & =R\left(X, X^{\prime}\right) \exp \left[-\frac{1}{2} U(X)+\frac{1}{2} U\left(X^{\prime}\right)\right], \\
l\left(X, X^{\prime}\right) & =L\left(X, X^{\prime}\right) \exp \left[-\frac{1}{2} U(X)+\frac{1}{2} U\left(X^{\prime}\right)\right] .
\end{aligned}
$$

They are each other's transpose, so the matrices $R, r, l$ and $L$ have the same eigenvalues.

The matrices under consideration consist of positive elements. By Perron's theorem (Bellman [13], Ch. 16.4) the absolutely greatest eigenvalue of such a matrix is positive and non-degenerate. The corresponding eigenvector can be chosen to consist of positive elements.

Let $\lambda$ be the largest eigenvalue of $R$ and $L$. Eqs. (14), (15), and (17) give then

$$
P=\frac{1}{n-1} \log \lambda \text {. }
$$

Because $L(\emptyset, \emptyset)=R(\emptyset, \emptyset)=1$, we have that $\lambda>1$, so $P>0$.

\section{The Equilibrium Condition}

In a previous paper [7], a number of equilibrium conditions have been proved to be equivalent. For the one dimensional lattice with interactions of range $n$, these conditions reduce to the very simple form $[4,7]$

$$
\mu_{\Lambda}(X)=\mu_{\Lambda}\left(X^{\prime}\right) \exp \left[-U(X)+U\left(X^{\prime}\right)\right]
$$


for any interval $\Lambda=[a, b]$, and any $X, X^{\prime}$ with

$$
\begin{aligned}
& X_{L} \equiv X \cap[a, a+n-2]=X_{L}^{\prime}, \\
& X_{R} \equiv X \cap[b-n+2, b]=X_{R}^{\prime} .
\end{aligned}
$$

Let us rewrite Eq. (20) in the form

$$
\mu_{\Lambda}(X)=p_{\Lambda}\left(X_{L}, X_{R}\right) \exp [-U(X)],
$$

where the $p_{A}\left(X_{L}, X_{R}\right)$ are non-negative numbers. We will show, that the consistency relations (1) uniquely determine the numbers $p_{A}$.

Theorem 1. The condition (20) uniquely determines the equilibrium state. The solution is

$$
\mu_{A}(X)=\mu_{\Lambda}(\emptyset) \psi_{L}\left(X_{L}\right) \psi_{R}\left(X_{R}\right) \exp [-U(X)],
$$

where $\psi_{L}$ and $\psi_{R}$ are the eigenvectors of the transfer matrices $L$ and $R$, corresponding to the largest eigenvalue. They are normalized according to

$$
\psi_{L}(\emptyset)=\psi_{R}(\emptyset)=1 .
$$

Proof. With any $\Lambda=[a, b]$, define

Write

$$
\begin{aligned}
\Lambda_{m} & =[a, b+m(n-1)] & & (m \geqq 0), \\
\Lambda_{-m} & =[a-m(n-1), b] & & (m \geqq 0) .
\end{aligned}
$$

where

$$
\begin{aligned}
\mu_{\Lambda_{m}}(X) & =p_{m}\left(Y_{0}, X_{m}\right) \exp [-U(X)], \\
\mu_{\Lambda_{-m}}(X) & =p_{-m}\left(Y_{m}, X_{0}\right) \exp [-U(X)],
\end{aligned}
$$

$$
\begin{aligned}
X_{m} & =X \cap[b+(m-1)(n-1)+1, b+m(n-1)], \\
Y_{m} & =X \cap[a-m(n-1), a-(m-1)(n-1)-1] .
\end{aligned}
$$

The consistency relations (1) yield

$$
\begin{aligned}
p_{m}\left(Y_{0}, X_{m}\right) \exp [-U(X)] & =\sum_{X_{m+1}} p_{m+1}\left(Y_{0}, X_{m+1}\right) \exp \left[-U\left(X \cup X_{m+1}\right)\right], \\
p_{-m}\left(Y_{m}, X_{0}\right) \exp [-U(X)] & =\sum_{Y_{m+1}} p_{-m-1}\left(Y_{m+1}, X_{0}\right) \exp \left[-U\left(Y_{m+1} \cup X\right)\right] .
\end{aligned}
$$

Using

we have

$$
\begin{aligned}
& U\left(X \cup X_{m+1}\right)-U(X)=U\left(X_{m} \cup X_{m+1}\right)-U\left(X_{m}\right), \\
& U\left(Y_{m+1} \cup X\right)-U(X)=U\left(Y_{m+1} \cup Y_{m}\right)-U\left(Y_{m}\right),
\end{aligned}
$$

$$
\begin{aligned}
p_{m}\left(Y_{0}, X_{m}\right) & =\sum_{X_{m+1}} R\left(X_{m}, X_{m+1}\right) p_{m+1}\left(Y_{0}, X_{m+1}\right), \\
p_{-m}\left(Y_{m}, X_{0}\right) & =\sum_{Y_{m+1}} L\left(Y_{m}, Y_{m+1}\right) p_{-m-1}\left(Y_{m+1}, X_{0}\right) .
\end{aligned}
$$


The transfer matrices $R$ and $L$ are defined in Eqs. (16) and (18). They consist of positive elements. Also the numbers $p_{m}$ must be nonnegative. Then, according to Lemma 2, which will be proved hereafter, the only solution of Eq. (24) is

$$
p_{0}\left(Y_{0}, X_{0}\right)=c \psi_{L}\left(Y_{0}\right) \psi_{R}\left(X_{0}\right),
$$

which proves the theorem.

We see, that the equilibrium condition (20) uniquely determines the equilibrium state. Remark, that no translational invariance has been imposed upon the state beforehand. The resulting state (23) proves to be invariant.

To conclude this section, we give the lemma used in the proof of Theorem 1.

Lemma 2. Let $L$ be a $N \times N$ matrix with positive elements. Let its greatest eigenvalue be $\lambda$, with the corresponding eigenvector $\psi$. Let $\left\{\varphi_{n}\right\}, n \geqq 0$, be a sequence of vectors with non-negative elements, such that

Then

$$
\varphi_{n}=L \varphi_{n+1}, \quad n \geqq 0 .
$$

$$
\varphi_{0}=c \psi \text {. }
$$

Proof. Let the transposed matrix $L^{\prime}$ have the eigenvector $\psi^{\prime}$ corresponding to $\lambda$. Choose the elements of $\psi$ and $\psi^{\prime}$ positive.

We have

$$
\left(\psi^{\prime}, \varphi_{0}\right)=\left(\psi^{\prime}, L^{n} \varphi_{n}\right)=\lambda^{n}\left(\psi^{\prime}, \varphi_{n}\right)=\lambda^{n} \sum_{k=1}^{N} \psi^{\prime}(k) \varphi_{n}(k) .
$$

Each term is non-negative, so for any $k$

$$
\varphi_{n}(k) \leqq \lambda^{-n}\left[\psi^{\prime}(k)\right]^{-1}\left(\psi^{\prime}, \varphi_{0}\right) .
$$

Also for any vector $\chi$

$$
\begin{aligned}
\left|\left(\chi, \varphi_{0}\right)\right| & =\left|\left(L^{\prime n} \chi, \varphi_{n}\right)\right| \\
& \leqq \lambda^{-n}\left(\psi^{\prime}, \varphi_{0}\right) \sum_{k=1}^{N}\left[\psi^{\prime}(k)\right]^{-1}\left|\left(L^{\prime n} \chi\right)(k)\right| .
\end{aligned}
$$

According to Bellman [13], Ch. 16.10,

$$
\lim _{n \rightarrow \infty} \lambda^{-n} L^{\prime n} \chi=\psi^{\prime}(\chi, \psi)\left[\left(\psi^{\prime}, \psi\right)\right]^{-1}
$$

for any vector $\chi$, so

$$
\left|\left(\chi, \varphi_{0}\right)\right| \leqq N\left(\psi^{\prime}, \varphi_{0}\right)|(\chi, \psi)|\left[\left(\psi^{\prime}, \psi\right)\right]^{-1} .
$$

In particular, if $\chi$ is orthogonal to $\psi, \chi$ is orthogonal to $\varphi_{0}$. That is,

This proves Lemma 2.

$$
\varphi_{0}=c \psi \text {. }
$$




\section{The Variational Principle}

Let us now formulate another way to come to equilibrium states. Given a translationally invariant state $\omega$, the mean entropy associated to it is defined as [14]

where

$$
s(\omega)=\lim _{\Lambda \rightarrow \infty}[N(\Lambda)]^{-1} S_{\Lambda}(\omega),
$$

$$
S_{\Lambda}(\omega)=-\sum_{X \subset A} \mu_{\Lambda}(X) \log \mu_{\Lambda}(X) .
$$

Now the pressure, corresponding to an interaction $\Phi$, Eqs. $(13,14)$, can also be found by [1]

$$
P(\Phi)=\sup _{\omega}\left[s(\omega)-\omega\left(A_{\Phi}\right)\right] .
$$

The supremum has to be taken over all translationally invariant states. Actually, the maximum is reached by a convex set of states. These states are defined to be equilibrium states.

In this section, we find once more the equilibrium state for the one dimensional lattice gas, now starting from Eqs. (25-27). The calculation is entirely different from the one in Section 4. It is somewhat more complicated, mainly, because translational invariance has to be imposed upon the state, which gives rise to the unhandy restrictions (6) and (7).

\subsection{The Mean Entropy}

The entropy for a one dimensional chain has first been studied by Mc Millan [15] in the context of information theory. An important result is the following.

Lemma 3. Let $\omega$ be any invariant state, and let

Then

$$
S_{n}(\omega)=-\sum_{X \subset[1, n]} \mu_{n}(X) \log \mu_{n}(X) .
$$

so

$$
0 \leqq S_{n+1}-S_{n} \leqq S_{n}-S_{n-1},
$$

$$
s(\omega)=\lim _{n \rightarrow \infty} n^{-1} S_{n}=\lim _{n \rightarrow \infty}\left(S_{n+1}-S_{n}\right) .
$$

Let us assume now that all $\mu_{n}(X)$ are given for some fixed $n$, and let them satisfy Eqs. (6). Then $S_{n}$ and $S_{n-1}$ are fixed, and for any invariant state with the given $\mu_{n}(X)$

$$
s(\omega) \leqq S_{n}-S_{n-1} .
$$

We have the following lemma. 
Lemma 4. Let the quantities $\mu_{n}(X)$ be given for some fixed $n$, and let them satisfy Eqs. (6). Then there is precisely one invariant state $\omega$, such that

$$
s(\omega)=S_{n}-S_{n-1} .
$$

The state $\omega$ is determined by (notation as in Eq. (12))

$$
\begin{aligned}
& \mu_{[1, k]}(X)=\mu_{[2, k]}\left(X^{(1)}\right) \mu_{[1, k-1]}\left(X^{(k)}\right)\left[\mu_{[2, k-1]}\left(X^{(1 k)}\right)\right]^{-1}, \\
& \text { if } \mu_{[2, k-1]}\left(X^{(1 k)}\right) \neq 0 \text {, } \\
& \mu_{[1, k]}(X)=0, \quad \text { if } \quad \mu_{[2, k-1]}\left(X^{(1 k)}\right)=0 \text {, }
\end{aligned}
$$

both relations for any $k \geqq n+1$.

Proof. The state $\omega$, defined by Eqs. (33), is indeed invariant. If the quantities $\mu_{k-1}(X)$ satisfy Eqs. (6), it follows from Eqs. (33) that the $\mu_{k}(X)$ do. Computing $S_{k}$ with Eqs. (28) and (33), one finds

$$
S_{k}=2 S_{k-1}-S_{k-2}
$$

for any $k \geqq n+1$, which yields Eq. (32).

The uniqueness of the state $\omega$ follows from the concavity of the entropy,

$$
S_{k}\left(\frac{1}{2} \omega_{1}+\frac{1}{2} \omega_{2}\right) \geqq \frac{1}{2} S_{k}\left(\omega_{1}\right)+\frac{1}{2} S_{k}\left(\omega_{2}\right),
$$

with the equality sign only for $\omega_{1}=\omega_{2}$.

\subsection{The Variation}

If the interaction has range $n$, the mean energy $\omega\left(A_{\Phi}\right)$ only depends on the quantities $\mu_{n}(X)$ (Eq. (12)). It follows, that a state $\omega$, that maximizes

$$
s(\omega)-\omega\left(A_{\Phi}\right),
$$

must satisfy Eqs. (33) for any $k \geqq n+1$. Further

$$
P=\max \left[S_{n}(\omega)-S_{n-1}(\omega)-\omega\left(A_{\Phi}\right)\right],
$$

where the maximum has to be taken over all sets $\left\{\mu_{n}(X)\right\}$ satisfying Eqs. (6). Let us first show that the maximum is reached for a unique set.

Lemma 5. The function

$$
\begin{gathered}
f\left(\left\{\mu_{n}(X)\right\}\right)=-\sum_{X \subset[1, n]} \mu_{n}(X) \log \mu_{n}(X) \\
+\sum_{X \subset[1, n-1]} \mu_{n-1}(X) \log \mu_{n-1}(X)-\sum_{X \subset[1, n]} \mu_{n}(X) B(X),
\end{gathered}
$$


where $B(X)$ is arbitrary, and $\mu_{n}(X)$ and $\mu_{n-1}(X)$ satisfy the consistency relations (7), reaches its maximum for a unique set $\left\{\bar{\mu}_{n}(X)\right\}$ with

$$
\bar{\mu}_{n}(X) \neq 0 \quad \text { for all } X \subset[1, n] .
$$

Proof. Let $\alpha$ of the numbers $\mu_{n}(X)$ and $\gamma$ of the numbers $\mu_{n-1}(X)$ equal 0 , with $\alpha \neq 0$.

Because of Eqs. (7),

Define

$$
\alpha \geqq 2 \gamma+1 .
$$

$$
\begin{aligned}
v_{n}(X) & =(1-\varepsilon) \mu_{n}(X)+\varepsilon 2^{-n}, \\
v_{n-1}(X) & =(1-\varepsilon) \mu_{n-1}(X)+\varepsilon 2^{-n+1} .
\end{aligned}
$$

Then also the quantities $v_{n}(X)$ and $v_{n-1}(X)$ satisfy Eqs. (7). Now

so

$$
f\left(\left\{v_{n}(X)\right\}\right)-f\left(\left\{\mu_{n}(X)\right\}\right)=(-\alpha+2 \gamma) 2^{-n} \varepsilon \log \varepsilon+\mathcal{O}(\varepsilon),
$$

$$
f\left(\left\{v_{n}(X)\right\}\right)>f\left(\left\{\mu_{n}(X)\right\}\right),
$$

if $\varepsilon$ is small enough. This proves that $f\left(\left\{\mu_{n}(X)\right\}\right)$ cannot be maximal if one of the $\mu_{n}(X)$ equals 0 .

The uniqueness of $\bar{\mu}_{n}(X)$ follows from the concavity of $f\left(\left\{\mu_{n}(X)\right\}\right)$. With Eqs. (7) and $\mu_{n}(X) \neq 0$, one shows directly that

and that

$$
\delta^{2} f\left(\left\{\mu_{n}(X)\right\}\right) \leqq 0
$$

$$
\delta^{2} f\left(\left\{\mu_{n}(X)\right\}\right)=0 \quad \text { only if } \delta \mu_{n}(X)=0 .
$$

This proves Lemma, 5 .

Let us now calculate the state that maximizes Eq. (34).

Theorem 6. Let the interaction $\Phi$ have range $n$. Then the equilibrium state is uniquely determined as follows. The quantities $\mu_{n}(X)$ and $\mu_{n-1}(X)$, and the parameters $V_{L}(X)$ and $V_{R}(X)$ follow from the Eqs. (7), together with

$$
\begin{aligned}
\mu_{n}(X) & =\mu_{n}(\emptyset) \exp \left[-U(X)-V_{L}\left(X^{(n)}\right)-V_{R}\left(X^{(1)}\right)\right], & & X \subset[1, n], \\
\mu_{n-1}(X) & =\mu_{n-1}(\emptyset) \exp \left[-U(X)-V_{L}(X)-V_{R}(X)\right], & & X \subset[1, n-1] .
\end{aligned}
$$

The quantities $\mu_{k}(X), k \geqq n-1$, are then

$$
\mu_{k}(X)=\mu_{k}(\emptyset) \exp \left[-U(X)-V_{L}\left(X_{L}\right)-V_{R}\left(X_{R}\right)\right],
$$

where $X_{L}=X \cap[1, n-1], X_{R}=X \cap[k-n+2, k]$.

Proof. We have to maximize Eq. (34) with the constraints (7). With the Lagrange multipliers $\lambda, \lambda^{\prime}, V_{L}(X)$ and $V_{R}(X)$, the quantity to be 
maximized becomes

$$
\begin{aligned}
& g\left(\left\{\mu_{n}(X)\right\},\left\{\mu_{n-1}(X)\right\}, \lambda, \lambda^{\prime},\left\{V_{L}(X)\right\},\left\{V_{R}(X)\right\}\right) \\
& =-\sum_{X \subset[1, n]} \mu_{n}(X) \log \mu_{n}(X)+\sum_{X \subset[1, n-1]} \mu_{n-1}(X) \log \mu_{n-1}(X) \\
& \quad-\sum_{X \subset[1, n]} \mu_{n}(X)\left[U(X)-\frac{1}{2} U\left(X^{(n)}\right)-\frac{1}{2} U\left(X^{(1)}\right)\right] \\
& \quad-\lambda\left[1-\sum_{X \subset[1, n]} \mu_{n}(X)\right]+\lambda^{\prime}\left[1-\sum_{X \subset[1, n-1]} \mu_{n-1}(X)\right] \\
& \quad+\sum_{X \subset[1, n-1]}\left[\mu_{n-1}(X)-\mu_{n}(X)-\mu_{n}(X \cup\{n\})\right]\left[V_{L}(X)+\frac{1}{2} U(X)\right] \\
& \quad+\sum_{X \subset[1, n-1]}\left[\mu_{n-1}(X)-\mu_{n}(X+1)-\mu_{n}(\{1\} \cup(X+1))\right]\left[V_{R}(X)+\frac{1}{2} U(X)\right] .
\end{aligned}
$$

The extra terms $\frac{1}{2} U(X)$ in the Lagrange multipliers have been introduced for convenience. Remark that $U(\emptyset)=0$, and that $V_{L}(\emptyset)$ and $V_{R}(\emptyset)$ may be chosen to be 0 , because the corresponding two equations follow from the remaining $2^{n}$ equations of (7).

Now

$$
\frac{\partial g}{\partial \mu_{n}(X)}=0, \quad \frac{\partial g}{\partial \mu_{n-1}(X)}=0
$$

give

$$
\begin{aligned}
1+\log \mu_{n}(X) & =-U(X)-V_{L}\left(X^{(n)}\right)-V_{R}\left(X^{(1)}\right)+\lambda, \\
1+\log \mu_{n-1}(X) & =-U(X)-V_{L}(X)-V_{R}(X)+\lambda^{\prime} .
\end{aligned}
$$

Substituting

$$
\lambda=1+\log \mu_{n}(\emptyset), \quad \lambda^{\prime}=1+\log \mu_{n-1}(\emptyset),
$$

one arrives at Eqs. (35).

To conclude the proof, suppose that Eq. (36) is satisfied for some pair $k-2, k-1$ with $k \geqq n+1$. Then Eqs. (33) give, that (36) is valid for $k$. To see that, one must realize that for $X \subset[1, k]$

$$
U(X)=U\left(X^{(1)}\right)+U\left(X^{(k)}\right)-U\left(X^{(1 k)}\right),
$$

because the interaction has range $n \leqq k-1$, and that

$$
X_{R}^{(1)}=X_{R}, \quad X_{L}^{(k)}=X_{L}, \quad X_{R}^{(1 k)}=X_{R}^{(k)}, \quad X_{L}^{(1 k)}=X_{L}^{(1)} .
$$

This proves Eq. (36) for all $k \geqq n-1$ by induction.

Let us now give the converse theorem.

Theorem 7. Let a translationally invariant state $\omega$ satisfy Eqs. (33) for $k \geqq n+1$, and let

$$
\mu_{n}(X) \neq 0 \quad \text { for any } X \subset[1, n] .
$$


Then there is a unique finite range interaction, the range of which is $n$, such that $\omega$ is the corresponding equilibrium state.

Proof. Take the logarithm of Eqs. (35), and consider the quantities $V_{R}(X), V_{L}(X)$ and $U(X)$ as unknown. One has $\left(3.2^{n-1}-2\right)$ linear equations with as many unknowns. In fact, there are $2\left(2^{n-1}-1\right)$ quantities $V_{L}(X)$ and $V_{R}(X)$, and $2^{n-1}$ potentials $\Phi(X)$. It remains to be proved that the homogeneous set of equations only has the trivial solution.

The homogeneous equations correspond to

$$
\tilde{\mu}_{n}(X)=2^{-n}, \quad \tilde{\mu}_{n-1}(X)=2^{-n+1} .
$$

Suppose that there is an interaction of range $n$, that gives $\tilde{\omega}$ as an equilibrium state. Eqs. (33) yield then

$$
\tilde{\mu}_{k}(X)=2^{-k}
$$

for any $k$. From Eq. (36) for $k=3 n-2$,

$$
U(X)+V_{L}\left(X_{L}\right)+V_{R}\left(X_{R}\right)=0
$$

for any $X \subset[1,3 n-2]$. If $X \subset[n, 2 n-1], X_{L}=X_{R}=\emptyset$, so

$$
\begin{array}{lll}
U(X)=0 & \text { for all } & X \subset[n, 2 n-1] ; \\
\Phi(X)=0 & \text { for all } X .
\end{array}
$$

Taking $X \subset[1, n-1]$ and $X \subset[2 n, 3 n-2]$, one finds that

$$
V_{L}(X)=V_{R}(X)=0
$$

for all $X$. This proves Theorem 7 .

\section{Conclusion}

The equilibrium state for a one dimensional lattice gas is found in two ways. The equilibrium condition, with the help of the matrix method, rather directly leads to the result. The variational method is more complicated. This arises from the fact, that translational invariance has to be imposed upon the state. The constraints, corresponding to this, in a natural way lead to the Lagrange multipliers $V_{L}(X)$ and $V_{R}(X)$. One may compare them with the temperature and the chemical potential, which occur as Lagrange multipliers if the entropy is maximized for given energy and density.

Comparison of the results in Eqs. (23) and (36) yields

$$
\psi_{L}(X)=\exp \left[-V_{L}(X)\right] ; \quad \psi_{R}(X)=\exp \left[-V_{R}(X)\right] .
$$


The restriction of the equilibrium state to a finite interval $[a, b]$ is a Gibbs state, where the energy of a configuration $X$ is taken to be (Eq. (36))

$$
U(X)+V_{L}\left(X_{L}\right)+V_{R}\left(X_{R}\right) .
$$

That is, on both ends $[a, a+n-1]$ and $[b-n+2, b]$ of the interval $[a, b]$ there are extra interactions, which are independent of the length of $[a, b]$. However these interactions do depend on the temperature and the chemical potential.

We also found (Theorem 7), that there is a one to one correspondence between the interaction of range $n$ and the invariant states with

$$
\mu_{n}(X) \neq 0, \text { and } s=S_{n}-S_{n-1} .
$$

The uniqueness of the equilibrium state, found here, implies, that it is the limit of finite volume Gibbs states. Generally, every limit of Gibbs states satisfies the equilibrium condition. The converse is not known (see e.g. [7], Section 7).

Let us make a remark on the Gallavotti-Miracle (KirkwoodSalsburg) equations [16] for the correlation functions $\varrho(X)$. They are proved $[4,7]$ to follow from the equilibrium condition. They have only one bounded solution if the temperature is high enough, thus giving rise to an upper bound for the critical temperature.

Now it is clear, that Eqs. (24) have more than one bounded solution, as soon as there are two or more eigenvalues of $L$ (and $R$ ) with absolute value $\geqq 1$. Then also the Gallavotti-Miracle equations have more than one bounded solution. Moreover, in that case the question remains open, whether the Gallavotti-Miracle equations are equivalent to the equilibrium condition.

There is a strong resemblence between the exact methods described in this paper, and the Bethe [17] approximation. Indeed, the Bethe approximation is known to be exact in one dimension.

Take, for example, a lattice where each point only interacts with its $\gamma$ nearest neighbours. With a lattice point $x$, take the volume

$$
\Lambda=\{x\} \cup \Lambda(x),
$$

where $\Lambda(x)$ is the set of nearest neighbours of $x$.

Then the equilibrium condition, Eq. (22), gives that

$$
\mu_{\Lambda}(X)=P\left(X_{1}\right) \exp [-U(X)],
$$

where $X_{1}=X \cap A(x)$. In the Bethe approximation, one simply takes

$$
P\left(X_{1}\right)=\mu_{\Lambda}(\emptyset) p^{N\left(X_{1}\right)}
$$

with a positive parameter $p$. If translational invariance is required for the state, one immediately arrives at the well known results $[17,18]$. 
The Bethe approximation is treated in a variational way by Hijmans and De Boer $[19,20]$. By combinatorial arguments, an expression for the mean entropy is found in terms of a few parameters. The difference of mean entropy and mean energy is then maximized with respect to these parameters.

In the simple example above, the mean entropy is approximated by

$$
s=\frac{1}{2} \gamma S_{2}-(\gamma-1) S_{1} .
$$

For $\gamma=2$ (one dimension), this reduces to the exact expression (32).

The methods used in the present paper for calculating the equilibrium state are typically one dimensional. In more dimensions, the most obvious generalization is the Bethe approximation. Exact solutions for two or more dimensional problems don't seem to have come closer.

Acknowledgements. The work described in this paper is part of the research program of the "Foundation for Fundamental Research on Matter" (F.O.M.), which is financially supported by the "Netherlands Organization for Pure Research" (Z.W.O). The author wishes to thank Professor N. M. Hugenholtz for his helpful discussions.

\section{References}

1. Ruelle, D.: Commun. math. Phys. 5, 324 (1967).

2. Haag, R., Hugenholtz, N. M., Winnink, M.: Commun. math. Phys. 5, 215 (1967).

3. Robinson, D. W.: Commun. math. Phys. 7, 337 (1968).

4. Lanford, O. E., Ruelle, D.: Commun. math. Phys. 13, 194 (1969).

5. Dobrushin, R. L.: Funkts. Analiz i ego Pril. 2, 31, 44 (1968).

6. Ginibre, J.: On some recent work of Dobrushin, Colloque International du C.N.R.S., Mai 1969, Gif-sur-Yvette.

7. Brascamp, H. J.: Commun. math. Phys. 18, 82 (1970).

8. Gallavotti, G., Miracle-Sole, S.: Commun. math. Phys. 5, 317 (1967).

9. Ruelle, D.: Statistical mechanics. New York-Amsterdam: Benjamin 1969.

10. - In:Fundamental problems in statistical mechanics, ed. E. G. D. Cohen. Amsterdam: North Holland 1968.

11. Kramers, H. A., Wannier, G. H.: Phys. Rev. 60, 252 (1941).

12. Baur, M., Nosanov, L.: J. Chem. Phys. 37, 153 (1962).

13. Bellman, R.: Introduction to matrix analysis. New York: Mc Graw-Hill 1960.

14. Robinson, D. W., Ruelle, D.: Commun. math. Phys. 5, 288 (1967).

15. Mc Millan, B.: Ann. math. Statistics 24, 196 (1953).

16. Gallavotti, G., Miracle-Sole, S.: Commun. math. Phys. 7, 274 (1968).

17. Bethe, H. A.: Proc. Roy. Soc. (London) A 150, 552 (1935).

18. Huang, K.: Statistical mechanics, Chapter 16.4. New York: Wiley 1963.

19. Hijmans, J., de Boer, J.: Physica 21, 471, 485, 499 (1955).

20. - Thesis, Amsterdam (1956).

21. Ruelle, D.: Commun. math. Phys. 9, 267 (1968).

H. J. Brascamp

Institute for Theoretical Physics

Rijks-Universiteit

P.O. Box 800,

Groningen, Netherlands 